\title{
Avaliação de fatores de risco para síndrome metabólica em grupo de funcionários de uma Escola Pública
}

\author{
Risk factors assessment for metabolic syndrome \\ in employee group of the Municipal School
}

Mayzza Campina Rodrigues ${ }^{\mathrm{I}}$ Evanuzia Miranda da Silva ${ }^{I I}$ Bhárbara Karolline Rodrigues SILVA ${ }^{I}$

Fernando Rodrigues Peixoto QUARESMA $^{1}$

Luis Fernando Castagnino SESTII

Fernando AdAmi ${ }^{1}$

Erika da Silva MacieL ${ }^{\text {III }}$

${ }^{1}$ Faculdade de Medicina do $\mathrm{ABC}$ (FMABC), Santo André/SP - Brasil.

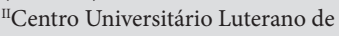
Palmas (CEULP/ULBRA), Palmas/ TO - Brasil.

"I'Universidade Federal do Tocantins, campus de Miracema do Tocantins TO - Brasil.
Resumo A Síndrome Metabólica é definida como um conjunto de alterações que aumentam os riscos de desenvolvimento de doenças crônicas não transmissíveis.

Objetivos: avaliar a presença de fatores de risco associado à síndrome metabólica em funcionários de uma escola pública de Palmas-TO.

Metodologia: Trata-se de um estudo de delineamento transversal, de natureza descritiva. As variáveis analisadas foram colesterol total, percentual de gordura, glicemia em jejum, triglicérides, pressão arterial sistólica e diastólica. O perímetro abdominal e o índice de massa corporal foram classificados de acordo com os critérios da Organização Mundial da Saúde. Para a classificação de Síndrome Metabólica (SM) foram adotados os parâmetros do NCEP-ATPIII (National Cholesterol Education Program's Adult Treatment Panel III), para o nível de atividade física, foi utilizado o Questionário Internacional de Atividade Física (IPAQ), versão 8. A análise de dados foi realizada de forma descritiva e empregado os testes de comparação entre os fatores de risco nas faixas de classificações do índice de Massa Corporal (IMC).

Resultados: Participaram da pesquisa 26 voluntários, entre os resultados destacam-se que $88,5 \%$ da população apresenta percentual de gordura elevado. Os critérios do NCEP-ATPIII caracterizaram SM em 19,2\%. Para os indivíduos classificados com sobrepeso ou obesidade, todas as demais variáveis mostraram valores superiores àqueles classificados como eutróficos. A análise estatística indicou que há diferenças entre os grupos com significância nos indicadores de composição corporal, sendo o conjunto dessas variáveis o principal fator de risco. Conclusão: $\mathrm{O}$ sobrepeso e a obesidade foram os fatores de risco que mais se destacaram no grupo estudado. Medidas preventivas relacionadas ao estilo de vida podem contribuir para evitar a instalação da SM.

Palavras chaves: Obesidade. Doenças Cardiovasculares. EstiLO DE Vida SEdentário. Atividade Física. 
Aвstract The Metabolic Syndrome is defined as a set of changes that increase the risks of developing chronic noncommunicable diseases.

Objectives: to evaluate the presence of risk factors associated to the metabolic syndrome in employees of a public school in Palmas-TO.

Methodology: This is a cross-sectional, descriptive study. The variables analyzed were total cholesterol, fat percentage, fasting glucose, triglycerides, systolic and diastolic blood pressure. Abdominal perimeter and body mass index were classified according to World Health Organization criteria. The National Cholesterol Education Program's Adult Treatment Panel III (NCEP-ATPIII) parameters were used to classify Metabolic Syndrome (MS). The physical activity level used the International Physical Activity Questionnaire (IPAQ), version 8. The data analysis was performed in a descriptive way and used the tests of comparison between risk factors in the ranges of the Body Mass Index BMI).

Results: Twenty-six volunteers participated in the study, among the results, $88.5 \%$ of the population had high fat percentage. The NCEP-ATPIII criteria characterized SM in 19.2\%. For individuals classified as overweight or obese, all other variables presented values higher than those classified as eutrophic. The statistical analysis indicated that there are differences between the groups with significance in the indicators of body composition being the set of these variables the main risk factor. Conclusion: Overweight and obesity were the most important risk factors in the studied group. Preventive measures related to lifestyle can contribute to avoid the establishment of MS.

Key-words: Obesity. Cardiovascular diseases. Sedentary lifestyLe. Physical activity.

\section{INTRODUÇÃO}

A Síndrome Metabólica (SM) é definida como um conjunto de fatores de risco que aumentam as chances de desenvolver Doenças Crônicas Não Transmissíveis (DCNT), entre essas, destacam-se as doenças cardiovasculares, resistência à insulina e complicações decorrentes. ${ }^{1}$

Os critérios da SM são determinados pela obesidade abdominal, hiperglicemia, triglicérides alterados, baixos índices de lipoproteína de alta densidade (HDL) e a hipertensão arterial. Entre esses, a obesidade abdominal tem sido destacada como o principal fator desencadeador das demais alterações metabólicas, ${ }^{1,2}$ pois aumenta o risco de morbimortalidade decorrentes dos fatores de risco cardiovasculares envolvidos na SM.
O excesso de peso está relacionado ao desenvolvimento de desordens fisiológicas e metabólicas, como a presença de hipertensão arterial, alterações no perfil lipídico e hiperinsulinemia, condições essas que, além de estarem associadas à SM, também estão relacionadas com o desenvolvimento de doenças cardiovasculares e diabetes melittus tipo 2.,4

As mudanças no estilo de vida e o sedentarismo são fatores desencadeantes para o surgimento da obesidade que, como consequência, elevam o risco de mortalidade provocada pelas DNCT em $20 \%$ a $30 \% .^{5}$

Os fatores de risco para SM tendem ser mais frequentes na fase adulta/idosa e a prática de exercícios físicos regularmente pode ser um aliado à prevenção das alterações metabólicas. ${ }^{1}$ 
Dessa forma, identificar e acompanhar os fatores de risco para SM é importante, tendo em vista o crescimento da associação com potenciais efeitos deletérios à saúde e a repercussões negativas na qualidade de vida, além de ser um atual problema de saúde pública. ${ }^{6}$

Tendo em vista que a SM é um distúrbio que está relacionado com as DCNT, as quais são responsáveis por um crescente número de óbitos nas últimas décadas no Brasil e no mundo, os seus fatores de risco geralmente são associados porém, silenciosos, e a presença de SM favorece o adoecimento das pessoas. ${ }^{7}$

Este estudo teve como objetivo avaliar os fatores de risco para a síndrome metabólica no quadro de funcionários de uma Escola Pública Municipal no Brasil.

\section{MATERIAIS E MÉTODOS}

\section{Tipo de estudo}

Trata-se de um estudo transversal, analítico descritivo. Realizado com funcionários de uma escola pública, no primeiro semestre de 2015. Foram convidados a participar da pesquisa todos os profissionais da escola (50 indivíduos), de forma voluntária.

\section{Critérios de Elegibilidade}

Foram incluídos na pesquisa todos os funcionários que estavam alocados na escola pública do município de Palmas, capital do Estado do Tocantins, norte do Brasil. Foram excluídos os profissionais que estavam de férias, os que se recusaram a assinar o Termo de Consentimento Livre e Esclarecido (TCLE) e outliers em relação aos dados bioquímicos.

\section{Aspectos Éticos}

$\mathrm{O}$ projeto foi submetido e aprovado pelo Comitê de Ética em pesquisa com Seres Humanos (CEP), sob o número CAAE 07564412.0.0000.5516.

\section{INSTRUMENTOS DE COLETA DE DADOS}

\section{Dados Socioeconômico demográfico}

Para analisar os dados econômicos, foi utilizado o Questionário de Critério de Classificação Econômica do Brasil. ${ }^{8}$ Concomitantemente, foram acrescidas perguntas de cunho descritivo para a caracterização dos participantes, com as variáveis sexo e idade.

\section{Composição corporal}

Para a avaliação do perímetro abdominal, foram adotados os critérios propostos pelo Instituto NCEP ATP III (National Cholesterol Education Program - Adult Treatment Panel III), que definem o ponto de corte de $102 \mathrm{~cm}$ para homens e $88 \mathrm{~cm}$ para mulheres. ${ }^{9}$

O peso e a altura foram verificados para realizar o cálculo do IMC, conforme os critérios da OMS, que seguem: baixo peso (IMC $\leq 18,5)$, faixa recomendável $(18,5 \leq$ IMC $\leq 24,9)$, sobrepeso $(25 \leq$ IMC $\leq 29,9)$, obesidade I $(30 \leq$ IMC $\leq 34,9)$, obesidade II $(35 \leq$ IMC $\leq 39,9)$ e obesidade III (IMC $\geq 40) .{ }^{10}$

A composição corporal foi medida por meio da análise da bioimpedância elétrica pelo Sistema Analisador Biodynamics Body Composition Analyzer 310 e produzido pela Biodynamics Corporation Seattle, Washing- 
ton USA. Esse sistema analisa os dados referentes à massa corporal, estatura, sexo e idade. $^{11}$

\section{Dados Bioquímicos}

Os indicadores bioquímicos foram obtidos por intermédio do aparelho Roche Accutrend Plus, por meio de teste rápido (ponta de dedo), que fornece resultados de triglicérides, colesterol total e glicemia em jejum (12h).

Os padrões de classificação para os indicadores bioquímicos desejáveis foram adotados de acordo com a V Diretriz Brasileira de Dislipidemias e Prevenção da Aterosclerose $^{12}$ valor para Triglicérides: $<150 \mathrm{mg} / \mathrm{dl}$, Colesterol Total: $<200 \mathrm{mg} / \mathrm{dl}$, e para Glicemia em jejum: $<100 \mathrm{mg} / \mathrm{dL}^{13}$

A pressão arterial foi avaliada utilizando o procedimento de aferição conforme a orientação da VII Diretriz Brasileira de Hipertensão Arterial, ${ }^{14}$ considerando os padrões de classificação do NCEP ATP III, ${ }^{9}$ que adota a seguinte classificação: sistêmica sistólica $\geq 130 \mathrm{mmHg}$ e diastólica $\geq 85$ $\mathrm{mmHg}$.

\section{Síndrome Metabólica}

Para a classificação da síndrome metabólica, foram utilizados os parâmetros NCEP-ATP III, que definem a presença de três dos cinco componentes adotados e que não distinguem etnia, tornando-os conveniente para a amostra estudada (Tabela 1). No entanto, essa definição preconiza uma alteração no ponto de corte para glicemia em jejum, $\geq 110 \mathrm{mg} / \mathrm{dL}$ para $\geq 100 \mathrm{mg} / \mathrm{dL}$, de acordo com alteração definida em 2005, sendo então denominado NCEP ATP III Revisado. ${ }^{9}$
Tabela 1 - Componentes da síndrome metabólica segundo o NCEP-ATP III.

\begin{tabular}{cc}
\hline Componentes & Níveis \\
\hline Obesidade & $>102 \mathrm{~cm}$ para homens \\
Abdominal & $>88 \mathrm{~cm}$ para mulheres \\
Triglicérides & $\geq 150 \mathrm{mg} / \mathrm{dL}$ \\
HDL Colesterol & $<40 \mathrm{mg} / \mathrm{dL}$ para homens \\
& $<50 \mathrm{mg} / \mathrm{dL}$ para mulheres \\
Pressão arterial & $\geq 130 \mathrm{mmHg}$ sistólica \\
Glicemia em & $\geq 85 \mathrm{mmHg}$ diastólica \\
jejum & $\geq 110 \mathrm{mg} / \mathrm{dL} / \geq 100 \mathrm{mg} / \mathrm{dL}$ \\
\hline
\end{tabular}

Fonte: Adaptado Rigo et al. ${ }^{15}$

Em relação ao colesterol, foi tomado como medida o colesterol total, tendo em vista que o aparelho utilizado não fornece os parâmetros fracionados.

\section{Nível de Atividade Física}

Para avaliar o nível de atividade física, foi utilizado o Questionário Internacional de Atividade Física (IPAQ), versão 8, forma curta e semanal. $\mathrm{O}$ questionário classifica o indivíduo em muito ativo, ativo, insuficientemente ativo e sedentário. ${ }^{16}$

\section{ANÁlise dos DADOS}

As variáveis qualitativas foram descritas por frequência absoluta e relativa. E as variáveis quantitativas por média, mediana, desvio padrão e os respectivos intervalos de confiança de $95 \%$.

Foi realizado o teste de Shapiro-Wilk para avaliar a aderência à distribuição normal das variáveis quantitativas. As variáveis colesterol, perímetro abdominal e pressão arterial sistólica mostraram aderência à distribuição normal dos dados, enquanto que as variáveis pressão arterial diastólica, triglicérides e glicose não apresentaram. Portan- 
to, para as variáveis quantitativas que apresentaram distribuição normal os resultados estão listados por média e as variáveis sem distribuição normal dos dados foram apresentadas por mediana.

Para analisar a associação entre os fatores associados à síndrome metabólica, foi utilizado o teste de Qui-quadrado.

Para fins de comparação entre as variáveis da composição corporal, foi utilizado o Teste de Anova e o Teste de Kruskal Wallis. Foi realizado o Teste Post HOC Bonferroni. O nível de confiança adotado foi de 95\%, o programa empregado foi o software Stata 11.0.

\section{RESUlTADOS}

Foram estudados 26 funcionários que atuavam na escola. A maior parte da amostra foi composta por indivíduos do sexo feminino (80,8\%), com idade média de 41,7 $( \pm 8,8)$ anos, com classificação econômica B1 (30,8\%), indivíduos que apresentaram alteração nos níveis de gordura corporal (88,5\%), pessoas que estão com IMC acima do recomendado (53,9\%) (Tabela 2).

Tabela 2 - Características dos participantes

\begin{tabular}{lcc}
\hline \multicolumn{1}{c}{ Características } & n & $\%$ \\
\hline Sexo & 21 & 80,8 \\
Feminino & 5 & 19,2 \\
Masculino & & \\
Classificação & & \\
socioeconômica & 7 & 26,9 \\
A & 8 & 30,8 \\
B1 & 7 & 26,9 \\
B2 & 3 & 11,5 \\
C1 & 1 & 3,9 \\
C2 & & \\
Percentual de gordura & 23 & 88,5 \\
Alterado & 3 & 11,5 \\
Normal & & \\
Índice de Massa & & 15,4 \\
Corporal & 4 & 38,5 \\
Obeso & 10 & 44,1 \\
Sobrepeso & 12 & IC $95 \%$ \\
Eutrófico & Média (dp) \\
& $41,7$ ( $\pm 8,8)$ & 38,$1 ;$ \\
Idade (anos) & 45,3 \\
\hline dp: desvio padrão. IC 95\%: Intervalo de confiança de $95 \%$
\end{tabular}


Em relação aos fatores de risco para a SM, 30,8\% dos participantes não apresentaram qualquer fator de risco enquanto que, $69,2 \%$ dos participantes revelaram um ou mais fatores de risco. O colesterol total $(38,5 \%)$ e o perímetro abdominal $(38,5 \%)$ foram os componentes com maior alteração (Tabela 3).

Tabela 3 - Prevalência de distúrbios metabólicos entre os participantes

\begin{tabular}{lccc}
\hline \multicolumn{1}{c}{ Distúrbios metabólicos } & n & \% & IC 95\% \\
\hline Triglicérides $\geq 150 \mathrm{mg} / \mathrm{dL}$ & 6 & 23,1 & 13,$9 ; 28,6$ \\
Colesterol Total $\geq 200 \mathrm{mg} / \mathrm{dl}$ & 10 & 38,5 & 34,$9 ; 71,5$ \\
Glicose $\geq 100 \mathrm{mg} / \mathrm{dL}$ & 2 & 7,7 & 6,$9 ; 14,3$ \\
Pressão Arterial Sistólica $\geq 130 \mathrm{mmHg}$ & 6 & 23,1 & 18,$6 ; 38,1$ \\
Pressão Arterial Diastólica $\geq 85 \mathrm{mmHg}$ & 1 & 3,9 & 2,$3 ; 4,7$ \\
Perímetro abdominal & & & \\
$>102 \mathrm{~cm}$ para homens & 10 & 38,5 & 25,$6 ; 52,4$ \\
$>88 \mathrm{~cm}$ para mulheres & & & \\
\hline
\end{tabular}

IC 95\%: Intervalo de confiança de 95\%.

Ao analisar o total de participantes com possível diagnóstico de SM, observa-se que 19,2\% estariam dentro dos critérios para a classificação adotados (Figura 1).

Figura 1 - Proporção de Síndrome Metabólica entre os participantes

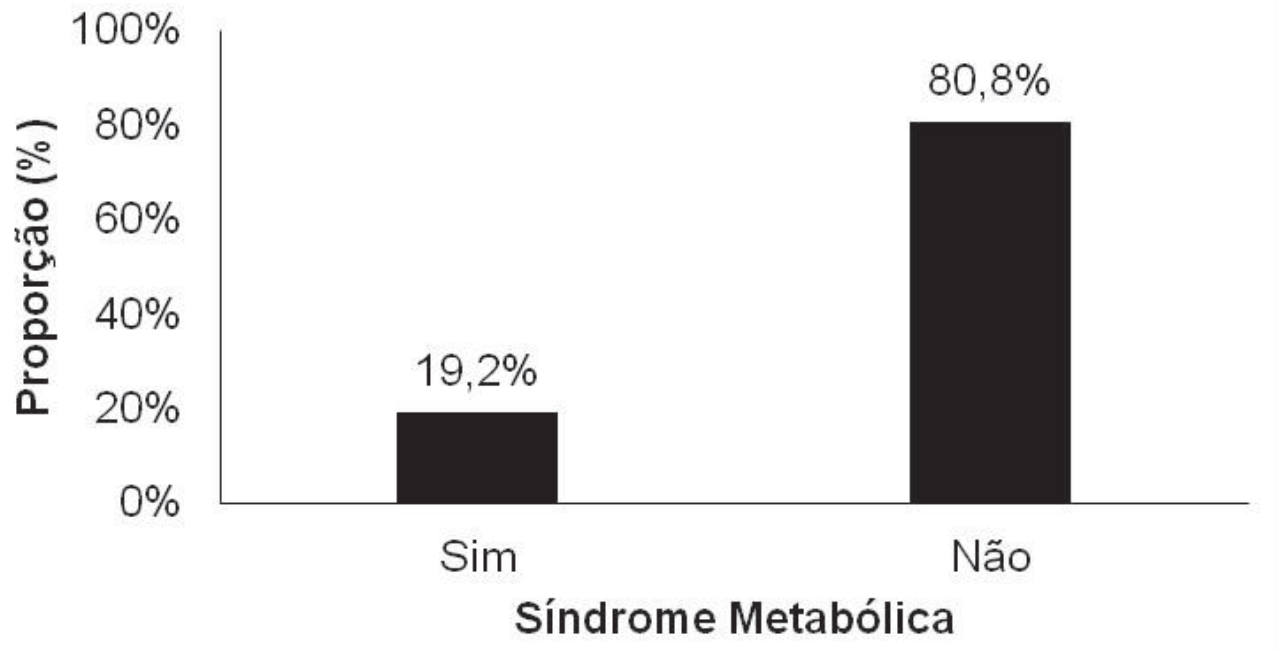


Foi realizado teste de Qui-Quadrado para avaliar a associação da SM com as demais variáveis. Entre as variáveis descritas, verificou-se que o diagnóstico de SM metabólica esteve presente apenas na população feminina. Além disso, o IMC mostrou diferença significativa entre os grupos de classificação $(\mathrm{p}<0,004)$, demonstrando que há associação positiva entre IMC e a presença de SM (Tabela 4 ).

Tabela 4 - Fatores associados à síndrome metabólica

\begin{tabular}{|c|c|c|c|}
\hline \multirow{3}{*}{ Fatores associados } & \multicolumn{2}{|c|}{ Síndrome Metabólica } & \multirow{3}{*}{$\mathbf{p}^{*}$} \\
\hline & Sim & Não & \\
\hline & \multicolumn{2}{|c|}{ n (\%) } & \\
\hline \multicolumn{4}{|l|}{ Sexo } \\
\hline Feminino & $5(23,8)$ & $16(76,2)$ & \multirow{2}{*}{0,225} \\
\hline Masculino & $0(0)$ & $5(100)$ & \\
\hline \multicolumn{4}{|l|}{ Classificação socioeconômica } \\
\hline A & $1(14,3)$ & $6(85,7)$ & \multirow{3}{*}{0,904} \\
\hline B & $3(20,0)$ & $12(80,0)$ & \\
\hline $\mathrm{C}$ & $1(25,0)$ & $3(75,0)$ & \\
\hline \multicolumn{4}{|l|}{ Percentual de Gordura } \\
\hline Alterado & $5(21,7)$ & $18(78,3)$ & \multirow{2}{*}{0,369} \\
\hline Normal & $0(0)$ & $5(100)$ & \\
\hline \multicolumn{4}{|l|}{ Índice de Massa Corporal } \\
\hline Obeso & $3(75,0)$ & $1(25,0)$ & \multirow{3}{*}{0,004} \\
\hline Sobrepeso & $2(20,0)$ & $8(80,0)$ & \\
\hline Eutrófico & $0(0)$ & $12(100)$ & \\
\hline
\end{tabular}

*Teste de Qui-quadrado.

Para comparar os fatores de risco para SM entre as classificações do IMC, foi realizado Teste de Anova e Kruskal-Wallis, tendo o IMC como variável preditora. Foram observadas diferenças estatisticamente significantes entre o perímetro abdominal $(p<0,001)$, em que os participantes classificados com obesidade e sobrepeso obtiveram os valores de perímetro abdominal superiores aos demais (Tabela 5). 
Tabela 5 - Comparativo entre classificações de IMC e os componentes da síndrome metabólica

\begin{tabular}{|c|c|c|c|c|}
\hline \multirow{3}{*}{ Variável } & \multicolumn{3}{|c|}{ Status nutricional } & \multirow{3}{*}{$\mathbf{p}^{*}$} \\
\hline & Obeso & Sobrepeso & Eutrófico & \\
\hline & \multicolumn{3}{|c|}{ Média (IC 95\%) } & \\
\hline Colesterol & $210(185,38 ; 234,61)$ & $190,77(156,08 ; 225,46)$ & $191,09(171,30 ; 210,87)$ & 0,616 \\
\hline $\begin{array}{l}\text { Perímetro } \\
\text { abdominal }\end{array}$ & $102,75(89,39 ; 116,10)$ & $91,11(87,49 ; 94,72)^{\mathrm{a}}$ & $78,73(75,09 ; 82,37)^{\mathrm{bc}}$ & $<0,001$ \\
\hline \multirow{2}{*}{$\begin{array}{l}\text { Pressão arterial } \\
\text { sistólica }\end{array}$} & $122,5(107,26 ; 137,73)$ & $123,0(111,29 ; 134,70)$ & $109,16(100,83 ; 117,49)$ & 0,067 \\
\hline & \multicolumn{3}{|c|}{ Mediana (IC 95\%) } & $\mathbf{p}^{\star *}$ \\
\hline $\begin{array}{l}\text { Pressão arterial } \\
\text { diastólica }\end{array}$ & $70(70 ; 70)$ & $70(63,24 ; 80)$ & $70(61,06 ; 70)$ & 0,760 \\
\hline Triglicérides & $105,5(98 ; 300)$ & $111,5(97,64 ; 203,76)$ & $104,5(93,53 ; 156,06)$ & 0,710 \\
\hline Glicose & $68,5(59 ; 178)$ & $61(46,56 ; 76,02)$ & $66(42,06 ; 70,57)$ & 0,565 \\
\hline
\end{tabular}

IMC: índice de massa corporal. ${ }^{\star}$ Teste de Anova. ${ }^{*}$ Teste de Kruskal-Wallis.

adiferença entre sobrepeso e obeso.

bdiferença entre normal e sobrepeso.

'diferença entre normal e obeso.

Em relação ao nível de atividade física, os indivíduos foram classificados como: sedentários 26,9\%, insuficientemente ativos $7,7 \%$, ativos $50 \%$ e muito ativos $15,4 \%$. Não houve associação entre o nível de atividade física e SM $(\mathrm{p}=0,442)$, porém, entre os indivíduos que apresentaram SM, foi possível verificar maior prevalência entre os insuficientemente ativos (50\%) e sedentários $(28,57 \%)$. Resultado que reforça a importância da atividade física como fator preventivo à SM.

\section{Discussão}

Os resultados do estudo reforçam que a alteração do IMC está associada com a SM e a obesidade central. Avaliada pelo perímetro abdominal, a obesidade foi o fator preponderante para a alteração do IMC.
Além disso, os resultados encontrados neste estudo são similares ao estudo na Ilha de São Miguel - Portugal, em que utilizaram os critérios da NCEP-ATP III e encontraram um quantitativo de $18,3 \%$ da amostra com fatores de risco para $\mathrm{SM},{ }^{2}$ esse percentual foi semelhante ao apresentado nos funcionários da escola pública deste estudo, em que $19,2 \%$ revelaram fatores de risco. Importante salientar que $69,2 \%$ do grupo apresentou pelo menos um fator de risco para SM.

Ao observar alguns fatores de risco de maneira isolada, obtêm-se dados preocupantes, como o caso dos indivíduos com sobrepeso e obesos (53,9\%). Esses indicadores são classificados como os principais fatores de risco, que trazem como consequências os distúrbios metabólicos que quando somados classificam a presença de SM. ${ }^{17}$

O excesso de peso e o sedentarismo de- 
notam que a relação gasto e consumo energético está em desequilíbrio, ${ }^{2}$ o que corrobora com os resultados encontrados na pesquisa, em que foi observada maior prevalência de SM entre os insuficientemente ativos (50\%) e sedentários (28,57\%). O trabalho de Leitão e Martins ${ }^{18}$ demonstrou que o sedentarismo e o baixo nível de atividade física aumentam significativamente a chance da população desenvolver SM, quando comparado aos níveis de atividade física moderada e intensa.

A atividade física é fundamental para o gasto energético e a manutenção do controle do peso, ajuda na redução da pressão arterial, eleva o HDL e melhora o controle glicêmico. ${ }^{19}$ Os benefícios da prática regular de exercício físico podem auxiliar nessa realidade. A atividade física é indicada como uma forma de promoção e prevenção de doenças, evitando sua progressão e contribuindo com uma melhor qualidade de vida. ${ }^{5}$

$\mathrm{O}$ indivíduo que se mantém acima do peso pode ter o perímetro abdominal aumentado. Este é um dos indicadores usados por todas as instituições que diagnosticam a SM como um fator de risco. Neste estudo, encontrou-se um total de 38,5\% dos indivíduos, sendo $100 \%$ do sexo feminino, com perímetro abdominal aumentado. Leite e Anchieta ${ }^{17}$ realizaram um estudo com policiais civis do Distrito Federal em que o percentual encontrado de indivíduos do sexo feminino (68\%) com perímetro abdominal aumentado foi bem maior que os do sexo masculino (45\%).

Entre os resultados, chama a atenção que os fatores de risco com maior prevalência com valores considerados acima da média foram: o percentual de gordura ele- vado $(88,5 \%)$, em segundo pelos índices de sobrepeso e obesidade $(53,9 \%)$ e o CT e perímetro abdominal, ambos com $38,5 \%$. A variável IMC reflete a coerência dos indicadores de composição corporal como aliado ao diagnóstico da SM. O fator com menor prevalência foi a $\mathrm{PAD}$, ao contrário dos resultados obtidos por Santos et al. ${ }^{2}$ no qual a glicemia foi o fator de risco com menor incidência e a pressão arterial foi o fator de maior incidência.

Os hábitos alimentares da população brasileira têm-se caracterizado por um aumento no consumo de alimentos industrializados, ricos em gorduras, açúcar simples e sódio e pobres em fibras. ${ }^{20}$ As modificações do padrão nutricional da população são confirmadas pelo aumento de sobrepesos e obesos e diminuição da desnutrição. ${ }^{21}$ Os novos hábitos alimentares associados à alimentação servida para os funcionários na merenda escolar, rica em carboidratos, corroboram com o índice elevado de indivíduos acima do peso encontrados nesta pesquisa.

Realizar refeições balanceadas, atendendo às necessidades energéticas e nutricional dos adultos, com a prática de atividade física, contribuirá para a redução do sobrepeso e obesidade, por conseguinte, auxiliando na redução desses fatores de risco para SM.

A alteração do perímetro abdominal foi estatisticamente significante entre os grupos de IMC. Isto reforça a ideia de que a alteração na composição corporal pode ser o indicador com maior potencial de alterações subsequentes, sendo o principal desencadeador dos distúrbios metabólicos.

Entre as limitações para estudos sobre SM, está o fato que as instituições como a 
OMS, a IDF e NCEP-ATP III apresentam pontos de corte distintos para SM, dificultando seu diagnóstico. ${ }^{14}$ Sendo o HDL Colesterol um dos fatores de análise para SM, a pesquisa traz como limitação a utilização de Colesterol Total, já que sua medição foi realizada por meio do aparelho Roche Accutrend Plus, que não fornece parâmetros fracionados.

\section{CONSIDERAÇões FINAIS}

Os resultados do presente estudo demonstraram que, embora a maioria dos participantes não apresente a SM instalada, há presença dos fatores de risco que podem evoluir para SM.

Nesse sentido, os resultados reforçam que a composição corporal parece o principal preditor para SM, seja por meio da classificação do IMC ou do perímetro abdominal elevado.

Soma-se a esses resultados o baixo nível de atividade física dos participantes, que contribui com o acúmulo de gordura, principalmente na região abdominal.

Dessa forma, sugere-se medidas de educação em saúde, como as ações de promoção e intervenção aos fatores de risco, estímulo a um estilo de vida mais ativo e práticas alimentares equilibradas.

\section{REFERÊNCIAS}

1. Faria ER de, Faria FR de, Franceschini S do CC, Peluzio M do CG, Sant'Ana LF da R, Novaes JF de, et al. Resistência à insulina e componentes da síndrome metabólica, análise por sexo e por fase da adolescência. Arq Bras Endocrinol Metabol. 2014;58(6):610-8.

2. Santos R, Nunes A, Ribeiro JC, Santos P, Duarte JAR, Mota J. Obesidade, Síndrome Metabólica e Atividade Física: Estudo exploratório realizado com adultos de ambos os sexos, da Ilha de S. Miguel, Região Autônoma dos Açores, Portugal. Rvista Bras Educ Física e Esporte. 2005;317-28.

3. De Oliveira CL, De Mello MT, Cintra IDP, Fisberg M. Obesidade e síndrome metabólica na infância e adolescência. Rev Nutr. 2004;17(2):237-45.

4. Weiss R, Dziura J, et al. Obesity and the Metabolic Syndrome in Children and Adolescents. N Engl J Med. 2004;2362-74.

5. Bernardes LE, Vieira EES, Lima LH de O, Carvalho GCN, Silva ARV da. \&lt;b\&gt; Fatores de risco para doenças crônicas não transmissíveis em universitários/ Risk factors for chronic noncommunicable diseases in university students\&lt;b\&gt; Ciência, Cuid e Saúde [Internet]. 2015;14(2):1115. Available from: http://periodicos.uem.br/ojs/index.php/CiencCuidSaude/article/view/22517

6. Pinho PM De, Maria L, Machado M, Torres RDS, Moura SE. Síndrome metabólica e sua relação com escores de risco cardiovascular em adultos com doenças crônicas não transmissíveis. Rev da Soc Bras Clínica Médica. 2014;12(1):22-30.

7. Duncan BB, Chor D, Aquino EML, Bensenor IM. Doenças crônicas não transmissíveis no Brasil : prioridade para enfrentamento e investigação Transmissíveis no Brasil : prioridade para enfrentamento e investigação Chronic Non-Communicable Diseases in Brazil : priorities for disease management and resear. Rev Saúde Pública. 2012;46:126-34.

8. Associação Brasileira de Empresas de Pesquisa. Critério Brasil 2015 e atualização da distribuição de classes para 2016. Critério Classif econômica Bras [Internet]. 2016;1-6. Available from: http://www.abep.org/criterio-brasil 
9. Grundy SM, Cleeman JI, Daniels SR, Donato KA, Eckel RH, Franklin BA, et al. Diagnosis and management of the metabolic syndrome: An American Heart Association/National Heart, Lung, and Blood Institute scientific statement. Circulation. 2005;112(17):2735-52.

10. Birdthistle I, World Health Organization. Dept. of Health Promotion/NCD Prevention and Surveillance Birdthistle IWHOD of HPP and S. Improving Health Through Schools National and International Strategies School. 2000;124.

11. Internacional V V. MONITOR DE COMPOSIÇÃO CORPORAL BIODYNAMICS MODELO 450 Os relatórios impressos por este monitor não são prescrições médicas . Ao explicar os resultados para seus pacientes ou alunos, aconselhe o seguinte: "Procure o seu médico antes de iniciar qualquer . :1-16.

12. Xavier H t., Izar MC, Faria Neto JR, Assad MH, Rocha VZ, Sposito AC. V DIRETRIZ BRASILEIRA DE DA ATEROSCLEROSE V D iretriz B rasileira de D islipidemias e P revenção. Arq Bras Cardiol. 2013;101(4, supl.1):1-20.

13. Lyra R, Oliveira M, Lins D, Cavalcanti N, Gross JL, Maia FFR, et al. Diretrizes Sociedade Brasileira de Diabetes [Internet]. Vol. 5, Diabetes Mellitus Tipo 1 E Tipo2. 2016. 709-717 p. Available from: http://bvsms.saude.gov.br/bvs/publicacoes/diabetes_mellitus.PDF\%255Cnhttp://www. scielo.br/scielo.php?script=sci_arttext\&pid=S0021-75572002000300004\&lng=en\&nrm=iso\&tl $\mathrm{ng}=\mathrm{pt}$

14. Sociedade Brasileira de Cardiologia. VII Diretriz Brasileira De Hipertensão Arterial. Arq Bras Cardiol [Internet]. 2016;107(3):1-83. Available from: www.arquivosonline.com.br

15. Rigo JC, Vieira JL, Dalacorte RR, Reichert CL. Prevalência de síndrome metabólica em idosos de uma comunidade: comparação entre três métodos diagnósticos. Arq Bras Cardiol [Internet]. 2009;85-91. Available from: http://www.scielo.br/scielo.php?script=sci_arttext\&pid=S0066$-782 \mathrm{X} 2009000800004 \&$ lang $=\mathrm{pt}$

16. Matsudo SM, Matsudo VR, Araújo T, Andrade D, Andrade E, Oliveira L. Nível de atividade física da população do Estado de São Paulo: análise de acordo com o gênero, idade , nível socioeconômico, distribuição geográfica e de conhecimento. Rev Bras Ciência e Mov. 2002;10(4):2002.

17. Leite, Eliziane Brandão; Anchieta VCC. Identificação de síndrome metabólica em policiais civis do Distrito Federal , Brasil. Brasília Med. 2013;50(3):186-93.

18. Leitão MPC, Martins IS. Prevalência e fatores associados à síndrome metabólica em usuários de unidades básicas de saúde em São Paulo - SP. Rev Assoc Med Bras [Internet]. 2012;58(1):609. Available from: http://www.scielo.br/scielo.php?script=sci_arttext\&pid=S0104$-42302012000100016 \&$ lang=pt\%250Ahttp://www.scielo.br/pdf/ramb/v58n1/v58n1a16.pdf

19. Sociedade Brasileira de Cardiologia. I Diretriz Brasileira de Diagnóstico e Tratamento da Síndrome Metabólica. Arq Bras Cardiol. 2005;84(suplemento I):4-28.

20. Molena-Fernandes CA, Junior NN, Tasca RS, Pelloso SM, Kenji R, Cuman N. A importância da associação de dieta e de atividade física na prevenção e controle do Diabetes mellitus tipo 2. Acta Sci Heal Sci Mar. 2005;27(2):195-205.

21. Gusmão LS, Sperandio N, Morais DC De, Dutra LV, Pinto A, Priore SE. Utilização de indicadores dietéticos como critérios prognósticos da Síndrome Metabólica Use of dietary indicators as prognostic criteria of the Metabolic Syndrome. 2014;7527:37-46. 


\section{DADOS DOS AUTORES}

\section{MAYZZA CAMPINA RODRIGUES}

Mestranda em Ciências da Saúde na Faculdade de Medicina do ABC. Enfermeira concursada do Hospital Geral de Palmas Doutor Francisco Ayres. Palmas/TO - Brasil. mayzza. rodrigues@gmail.com

\section{EVANUZIA MIRANDA DA SILVA}

Mestranda em Engenharia Ambiental na Universidade Federal do Tocantins. Participa do grupo de pesquisa Núcleo de Estudos em Educação Ambiental do Instituto Federal do Tocantins. Palmas/TO - Brasil. evanuzia@gmail.com

\section{BHÁRBARA KAROLLINE RODRIGUES SILVA}

Mestranda em Ciências da Saúde na Faculdade de Medicina do ABC. Membro do Laboratório de Epidemiologia e Análise de dados da Faculdade de Medicina do ABC. Santo André/SP - Brasil. bharbarakarolline@gmail.com

\section{FERNANDO RODRIGUES PEIXOTO QUARESMA}

Doutorando em Ciências da Saúde na Faculdade de Medicina do ABC. Docente do Centro Universitário Luterano de Palmas e da Universidade Federal do Tocantins. Palmas/TO Brasil.ferodriguesto@gmail.com

\section{LUIS FERNANDO CASTAGNINO SESTI}

Doutorando em Biologia Celular e Molecular Aplicada a Saúde na Universidade Luterana do Brasil. Docente do Centro Universitário Luterano de Palmas. Palmas/TO - Brasil. luis. sesti@ceulp.edu.br

\section{FERNANDO ADAMI}

Doutor em Epidemiologia pela Universidade de São Paulo. Docente e Coordenador do Laboratório de Análise de Dados na Faculdade de Medicina do ABC. Santo André/SP - Brasil. adamifernando@uol.com.br

\section{ERIKA DA SILVA MACIEL}

Doutora em qualidade de alimentos pela Universidade de São Paulo. Docente da Universidade Federal do Tocantins. Araguaína/TO - Brasil. erikasmaciel@gmail.com

Submetido em: 6-7-2017

Aceito em: 19-10-2017 